\title{
A healing-centered approach to preventing urban gun violence: The Advance Peace Model
}

Jason Corburn (10 ${ }^{\mathbb{}}$, DeVone Boggan², Khaalid Muttaqi ${ }^{2}$, Sam Vaughn ${ }^{3}$, James Houston ${ }^{3}$, Julius Thibodeaux ${ }^{4} \&$ Brian Muhammad ${ }^{5}$

Urban gun violence is the result of and contributes to trauma for both individuals and communities. In the US, African American males between 15 and 34 years old bear the greatest mortality burden from gun violence. Community-based approaches that use credible, street-level outreach workers to interrupt conflicts, mentor the small number of offenders in each community, and offer them alternatives to violent conflict resolution, have demonstrated success in reducing firearm homicides. Yet, few of these approaches explicitly aim to also address the traumas of structural violence that contribute to gun crime, including dehumanizing policing, extreme poverty, and institutional racism. This commentary describes a program called Advance Peace that aims to explicitly use a healing-centered approach to address the traumas associated with violence as a means to reduce gun crime in urban communities. We describe the traumainformed, healing-centered approach used by Advance Peace, the components of its intensive outreach strategy called the Peacemaker Fellowship, and some impacts the program is having on trauma and healing. The evidence comes from observations, interviews, and the voices of Advance Peace participants and staff. We suggest that exploring the inner workings of the Advance Peace model is critical for identifying ways to support trauma-informed healing-centered approaches in Black and brown communities that have been ravaged by racism, incarceration, and heavy-handed state violence.

\footnotetext{
${ }^{1}$ School of Public Health \& Department of City \& Regional Planning, UC Berkeley, Berkeley, CA, USA. ${ }^{2}$ Advance Peace, Richmond, USA. ${ }^{3}$ Office of Neighborhood Safety, Richmond, CA, USA. ${ }^{4}$ Advance Peace Sacramento, Sacramento, USA. ${ }^{5}$ Advance Peace Stockton, Stockton, USA.

凶email: jcorburn@berkeley.edu
} 


\section{Introduction}

rban gun violence is public health, policy, and development issue. In cities across the US, young Black men are most likely to be both victims and perpetrators of gun violence (Abt and Winship, 2016; Kalesan et al., 2019; Rivera et al., 2018; GBD, 2016). Yet, increasing evidence suggests that entire communities are not violent, but there are a very small number of individuals who are driving disproportionate amounts of urban gun violence (Papachristos and Wildeman, 2014). The traumas from state-sanction violence, including heavy-handed policing, racially segregated neighborhoods, displacement, and lack of educational and economic opportunities, combine to contribute to urban gun violence and victimization, and many people in poor, communities of color do not trust the state or justice system (Akbar, 2017; Francis, 2018; Fullilove, 2004; Geller et al., 2014; Kern, 2016). According to the US Center for Disease Control and Prevention (CDC) web-based injury statistics query reporting system (WISQRS), in 2018 the firearm homicide rate per 100,000 persons for Black males was 37.7 compared to 21 for all Blacks and 4.5 for the entire population. Yet, these same data reveal that for Black Males 15-34 years old, the firearm homicide rate in 2019 was $81.5 / 100,000$ persons, $117 \%$ greater than just for all Black males (CDC, 2020).

This commentary argues that urban gun violence reduction policy and practice must recognize and address the traumas of violence, not just aim to reduce rates of crime, by focusing on healing the small number of potentially violent perpetrators at the center of most gun violence. While some community-based gun violence programs, such as Cease Fire and Cure Violence (CV), do focus on the small number of offenders and have demonstrated success in reducing gun crime, these programs rarely if ever report on whether or not the participants or communities are healing from decades of dehumanization, trauma, racism and other forms of structural violence (Braga et al., 2019; Butts et al., 2015; Friedson and Sharkey, 2015; Garvin et al., 2013; PicardFritsche and Cerniglia, 2013; Ranney et al., 2019; Skogan et al., 2008). We ask whether a new community-driven urban gun violence prevention program called Advance Peace (AP) might offer insights for a trauma-informed, healing-centered approach to addressing urban violence and community health (Motlagh, 2016; Rani, 2017; Wolf et al., 2015).

The AP model has been used in Richmond, California, and a 2019 study revealed that this program contributed to a 55\% reduction in gun homicides and assaults (Matthay et al., 2019). In 2021, AP was operating in Richmond, Sacramento, Stockton and Fresno, California, Ft. Worth, Texas, and was being considered by at least 12 other cities in the US and around the world (www. advancepeace.org). We describe the frameworks informing the AP model, use the voices of participants (some of whom are coauthors), and describe the AP Peacemaker Fellowship-its 18month curriculum that utilizes street-outreach workers to deliver everyday adult mentorship, group life-skills classes, social services, and other healing-centered supports to those at the center of urban gun violence. We describe how AP implements their healingcentered approach and suggest that studying the AP model can offer governments ideas for 'justice investments' into urban communities that have experienced dehumanizing policing, mass incarceration, and chronic divestment from life-supporting infrastructure. AP may offer urban communities a model that contributes to improved public safety as well as critically needed inter-generational healing, racial justice, and improved well-being (Norris, 2020; Sered, 2019).

\section{From trauma-informed to healing centered: addressing toxic stress}

The public health approach to gun violence reduction recognizes that (a) 'health' is a complete state of physical, mental, and social well-being, not merely the absence of disease (b) violence is a disease that affects the structure and function of the brain and causes morbidity and mortality, and (c) prevention demands stopping the 'transmission' and spread through a combination of behavioral and structural, meaning legal, policy and social norm, interventions (Decker et al., 2018). Building on the public health approach, trauma-informed strategies to address urban gun violence acknowledge the widespread impact that trauma has on youth deviance, that violence can be a reaction to unaddressed trauma, and addressing gun violence must not re-traumatize victims (SAMHSA, 2015). A prevention approach does not blame victims or perpetrators, but rather recognizes that young people who witness regular violence, whose peers are victims of violence, and who may be victims themselves, are most likely to engage in future violence (Pressley and Smith, 2017).

A trauma-informed approach begins by recognizing that violence is often an adaptive strategy for dealing with trauma (Lane et al., 2017). These traumas can be intergenerational and can range from childhood physical and sexual abuse, experiences with and witnessing violence, extreme deprivation, homelessness and poverty, racism, and/or living with a substance abuser or in a household with an incarcerated person (SAMHSA, 2015). These repeated traumas that happen early in life are called adverse childhood experiences (ACES), which adversely impact later-inlife decision making, cognitive abilities, and health outcomes (Danese and McEwen, 2012).

These and related traumas act as a form of toxic stress. Toxic stress results when there are frequent and sustained adverse life events, from an early age, without any buffering or support. Toxic stress results in the constant secretion of stress hormones, which cause internal inflammation to 'protect' against the perceived threats (Geronimus and Thompson, 2004). This constant state of alert results in a chronic "wear and tear" effect on multiple organ systems, compromising the immune system's ability to fight disease and the brain's development in areas such as self-esteem and impulse control (McEwen and McEwen, 2017). Many young people involved in gun crime have experienced what is called the 'pair of ACEs': such as emotional and physical neglect, sexual abuse, and exposure to domestic violence-and, Adverse Community Environments-such as racism, poverty, insecure housing, environmental pollution, heavy-handed policing, and prior exposure to gun violence (Ellis and Dietz, 2017).

AP argues for a shift from a trauma-informed to a healingcentered approach to gun violence reduction. As Ginwright (2018, p. 6) notes, a healing centered approach is "strengthsbased, advances a collective view of healing, and re-centers culture as a central feature in wellbeing," requiring a different set of questions that moves from 'what happened to you' to 'what's right with you?' The healing-centered approach also suggests that those who have experienced trauma and dehumanization participate in the active discovery of the causes of their harms, both inter-personal and within their communities (DeCou and Lynch, 2017). This 'ecologic approach' to healing involves identifying the historic public policies and social practices that have contributed to the harm, such as housing discrimination, workplace segregation, and dumping of toxic waste in communities of color (DeCandia and Guarino, 2015). Thus, a key feature of the healing-centered approach is active participation in one's healing, which can contribute to known wellness characteristics such as a sense of purpose and self-efficacy, self-esteem, and the power to control one's life situations (Gerber and Gerber, 2019).

\section{What is Advance Peace (AP)?}

$\mathrm{AP}$ is a community-based organization $(\mathrm{CBO})$ that identifies the most lethal individuals at the center of gun violence in a city, 
invests in them through 7-days-a-week mentoring and supportive relationships using street outreach workers, and delivers services and supports to these individuals during an 18-month program called the Peacemaker Fellowship (Lowery and Rich, 2018; Wolf et al., 2015). AP builds on the successful elements of tested programs such as CV (formerly known as Chicago Ceasefire), which uses a public health approach that focuses on those most at risk of 'spreading' gun violence and uses street outreach workers to change their behavior and attitudes. $\mathrm{CV}$ has demonstrated success in reducing shootings in cities such as New York, Chicago, and Philadelphia, by focusing on 'denormalizing' gang behaviors, using outreach workers to interrupt conflicts, and providing incentives to at-risk individuals to seek alternative models of conflict resolution (Butts et al., 2015). AP has important distinctions from $\mathrm{CV}$, including (a) AP participants do not have to be gang affiliated, while they do in CV, (b) AP does not affiliate with law enforcement, while $\mathrm{CV}$ works independently from police but builds close relations with them, (c) AP outreach workers are both interrupters of violence and mentors, while CV uses a separate staff for interruption and mentorship, (d) AP focuses on individual healing of trauma while $\mathrm{CV}$ includes individual and group norm change, (e) AP includes an intensive 18-month Fellowship program individualized for participants (described below) while CV uses anti-violence public events, education campaigns, and partnerships with other $\mathrm{CBO}$ s to build a social consensus against gun violence.

A key similarity between CV and AP is the employment of street-outreach workers from the communities where they work, all of whom have spent time in prison for gun crimes, but returned home with insights and skills for confronting their traumas and peacefully resolving conflicts (Chabria, 2018). AP calls these street-level credible messengers Neighborhood Change Agents (NCAs). As one interviewee stated:

You need people who have lived the life, but also seen the experiences comin' up that may have drove them to crime and violence. It's not just 'I've repented for some sins' but also, 'I'm aware of the shit I went through, it wasn't my fault, and I'm not to blame for what it did to me.'

Thus, the credible messengers within AP might be considered "wounded healers" (LeBel et al., 2015). The 'wounded healer' is someone that has experienced trauma, is often in the process of recovering, and uses those experiences to empathize and bond with others that have been similarly hurt. The wounded healer can not only empathize with those similarly hurt but also transforms their past into a source of wisdom to be drawn from while acting as a trusted mentor.

\section{The Peacemaker Fellowship}

The crux of AP's healing-centered approach is their Peacemaker Fellowship program. AP actively seeks to recruit the few hundred people in the community, generally $14-34$ years old, that is creating the risk of violence for everyone else, and aims to identify the $20-30$ people that are the most influential within that group. The AP outreach team will spend 6 or more months identifying and recruiting these highly influential individuals, and also recognize that these same individuals are also some of the most vulnerable since they are the most likely to be the victim of gun violence in their community. What makes the AP program unique is that they focus on the hardest to reach individuals, those who have been rejected from other organizations, and those that no other organization or institution wants to touch. Most of the future fellows have long rap sheets as teenagers which can deny them the ability to get back into school, a job and often gets them kicked out of their homes.

\section{Becoming a Fellow}

Once a trusting relationship is built between a fellow and their NCA, they work one-on-one to draft a Life Management Action Plan (LifeMAP). The LifeMAP is a key entry point into the fellowship, is tailored to the individual's needs and challenges, and kick-starts the 18-month intensive engagement. The LifeMAP sets short, medium, and long-term goals for personal safety, housing, education, employment, anger management, conflict resolution, creating positive social networks, financial literacy, behavioral/medical healthcare, substance use disorder support, parenting, recreation, and spirituality. Since the LifeMAP is tailored to the individual needs of each fellow, the NCA works closely with that person to really understand their life story and challenges, and the fellows are treated as assets, empowered to be active agents in their own success, and treated as the key ingredient to stopping gun violence in their own communities. The LifeMAP acts as both a mechanism for ensuring the fellow defines their own healing needs (i.e., 'for them, by them') and is the 'social contract' that a strong, caring, and consistent adult is willing to take a risk and believe in them. As one AP staff member put it: "We see each one of the fellows as the essential antidote to this urban epidemic of gun violence".

The LifeMAP becomes a 'living document' that guides mentorship but is also updated and adjusted to reflect new goals and the changing relationship between mentor and mentee. As one NCA noted:

These youngsters are acting out with guns it's really just a cry for help. But as a young man in our neighborhood you can't go to someone and say 'I'm hurting.' Its shamed or seen as weak. We have to be genuine. They can see if its fake. And invest in them when nobody has done that before. Let them know their worth, just for who they are, not anything they do or don't do.

Another aspect of the Fellowship, and built into most fellow's goals, is joining group learning sessions, or what AP calls 'lifeskills' classes. These 'classes' are more like facilitated dialogs, sometimes referred to in the healing literature as circles, where groups of fellows focus on a particular topic over a series of weeks or months. Typical healing 'life-skills' include discussions about what it means to be 'a man,' group Cognitive Behavioral Therapy (CBT), understanding the forces of structural racism, or how to be a good father/parent. Hardy (2013) notes that part of healing from racism involves recognizing the 'hidden wounds' of structural racism and ensuring responses challenge Euro-centric perspectives of racial trauma. The NCAs recognize that their skills only go so far and many fellows require additional professional supports. What tends to differentiate AP from other programs is that the NCA will not just refer their fellow to a service, but often physically take them there and accompany them to the organization. This is not only because fellows may be reluctant to seek services or be disillusioned after being let down by professionals before, but also because many service providers aren't prepared to handle the population that AP serves.

\section{Milestone allowance and transformative travel}

Each NCA keeps track of their weekly engagements, including the number of fellows and other street-level contacts, the hours spent, and the type of activities they perform, such as mediating a conflict, referring a fellow for services, or accompanying a fellow to a program or activity. This tracking helps document NCA investments in each fellow and the fellow's progress toward their LifeMAP goals. Once a fellow is working on their LifeMAP for at least 6 months and achieves $65 \%$ progress toward a goal (as determined by their NCA mentor and the AP leadership), that fellow is eligible for a milestone allowance of up to $\$ 1000$ USD per month. The exact 
amount of milestone allowance is based on effort and achievements. Importantly, the allowance is just one way to recognize the value of each fellow and signals to them that they are a community asset worth investing in and someone that has real value.

Another key offering of the Fellowship is what AP calls 'transformative travel.' This can occur after fellows have accomplished some short-term life-map goals, demonstrated to AP staff that they are committed to healing themselves, and building healthy relations with others. In most cases, fellows have never traveled outside their city and many rarely leave their neighborhoods. Group excursions can include community service projects, taking college tours, meeting with government officials, and participating in restorative justice dialogs in other communities. AP fellows are offered these privileged travel experiences on the condition that they travel with one of their rivals, who also happens to be a fellow.

A related 'transformational' aspect of the Fellowship is internships, where AP matches a fellow with a local employer and pays the Fellow's salary. As Fellows progress through the program, they also interact with an 'elder circle' or group of 'real OGs'. The elder circles can also act as spaces of healing for longsimmering conflicts and can show the younger fellows that the adults in their lives also need support and healing.

\section{Keeping us safe}

All the AP NCAs are skilled at mediating conflicts in the streets and in other settings. As 'violence interrupters' the NCAs regularly insert themselves in-between 'beefs' where guns are drawn and ready to be used. One NCA described how they mediate imminent gun conflicts and confront potential violence before police are called, stating:

Sometimes it's the girlfriend that will call us or another fellow. We might also hear about it from others on the streets or see a social media threat or a music video. We get that call and try to de-escalate the situation. Most critical is having a relationship with them, knowing something about their lives, what and who they care about. In every instance, somebody in the know eventually lets us know that we [AP NCA] was the determining factor for the standing down

Many street gun conflicts that NCAs mediate start as insults on social media, such as Facebook. AP NCAs spend at least a few hours every day scanning social media to review posts that might be interpreted as insults or lead to potential conflicts. NCAs will also attend to a family that has lost a loved one or when their child might be incarcerated. They might comfort family members, deliver food, or pay for a funeral.

Once a person has joined and even completed the Peacemaker Fellowship, they are considered a 'life-long' fellow, according to the AP leadership. This is in part because AP recognizes how hard it is to transition away from the life of gun violence and all your peer influencers. As the AP NCAs noted, their fellows struggle even if they want to leave the street life behind, since they are constantly being pulled back into their old ways, getting attacked and ridiculed for going down a different path. For this and other reasons, the NCAs remain supportive and in contact with their fellows even after the 18-month Fellowship, stating that "this isn't just a program for us or them, it's a way of life". The basic model of change, including activities, intermediate and long-term outcomes of the AP model are displayed in Fig. 1.

\section{Community-wide transformations}

Our descriptive findings combined with previously published results suggest that the AP program is working to heal individuals and communities. For example, in Sacramento gun homicides and assaults were reduced by $21 \%$ in the 18 -month fellowship from June 2018 through December 2019, compared to the average number of gun homicides and assaults from the same 18month period in the previous 4 years (Corburn and FututomeLopez, 2020). Sacramento also has not had a juvenile homicide in the 2 years since AP started to work in that city. In Stockton, during 2019, the first year of the Fellowship, AP NCAs helped prevent thirty-two murders through interrupting imminent gun conflicts, mediated 136 community conflicts that could have escalated into gun violence, and helped avoid further retaliatory violence by responding to 72 shootings (Corburn and Fukutome, 2019). In Richmond, California, 97\% of fellows who entered the program are still alive, $83 \%$ have not been injured by a firearm, and $66 \%$ have not been engaged in any new firearm activity (ONS, 2019).

During the intake of the fellows for the Peacemaker Fellowship (as part of the LifeMAP on-boarding), each fellow is asked a series of questions based on the Life Stressor Checklist-Revised instrument, which identifies whether or not (yes/no) each fellow had experienced a stressful event (Wolfe et al., 1997). Each fellow is tracked weekly by their assigned NCA, who documents any adverse life event (i.e., arrest, gun injury, etc.) and any services, counseling, and opportunities (i.e., travel, internships, employment, etc.) received. At the conclusion of the Fellowship, each fellow is asked about their orientation toward conflict resolution, their outlook on life, how stable and safe they feel in their lives, and questions about and how they would rate the Peacemaker Fellowship program itself. We present a subset of those questions for the 103 Fellows enrolled in the AP Sacramento program from 2018 to 2020 (Tables 1 and 2). These descriptive data suggest that the majority of fellows are indeed African-American males under 24 years old, the participants entered with a series of traumatic life events, and $84 \%$ were both previously incarcerated and had been the victim of a gunshot. These and related questions suggest a high degree of violencerelated trauma among the Fellows. At the conclusion of the Peacemaker Fellowship, some indicators of healing among the same cohort seem to be present. For example, almost all had received basic life-supporting assistance in the form of food and housing, $71.8 \%$ reported an improved outlook on life (i.e., hope), $84.2 \%$ reported having a trusting adult to talk to about a difficult life event, and $85.4 \%$ rated their AP outreach worker as one of the most important adults in their life.

While the broader impacts on community healing from the work of AP are difficult to determine, we found evidence of this from both governmental and community organizations stakeholders. A youth-focused $\mathrm{CBO}$ leader reflected on the post-AP community dynamic:

As the frequency of shootings went down, and our young people could ride the bus or walk home from school without fear, it was like a giant exhale. It's not perfect, but now more teens are riding bikes, using parks, just walking to the store. They just wouldn't do that before.

A safer place and a community where young people are invested in, brings an 'atmosphere of change,' as another CBO leader described the impact of AP. After the program, fellows are often more prepared to navigate social services, school, and other goods in their community that help them continue to grow and develop. An AP staff member described the community development and healing approach this way:

Our work is an ecosystem of love, healing, affirmation, harm reduction, investment of time, attention, services, opportunities, resources, personal and leadership 
Fellow Change

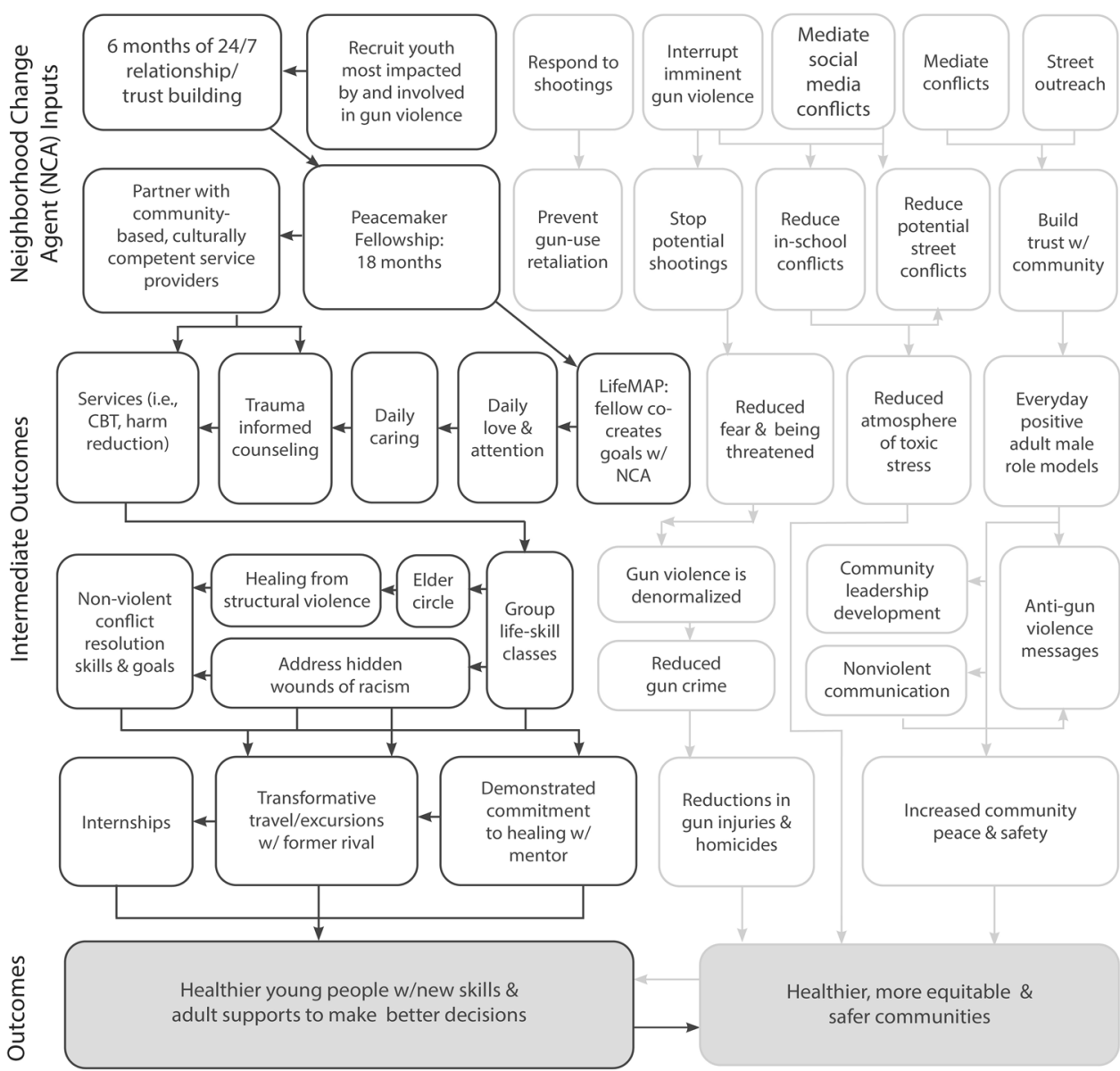

Fig. 1 The Advance Peace Model of Healing and Transformative Change. The left columns reveal the relationships between Advance Peace outreach worker supports, programs and services that combine to help heal individuals. The right columns reveal how these same and related program components support community healing and transformation.

\section{Table 1 Advance Peace Sacramento, fellow participant profiles at intake ${ }^{a}$.}

\section{Fellow characteristic}

\section{African American}

Male

Unemployed

Finished HS

Was/is in foster care system

Was/is/ever homeless

Prior gun arrest

Prior incarceration

Parent is/was incarcerated

Previous gunshot injury

Witness gun homicide

Ever physically assaulted

Someone close to you murdered

aNot all participants answered all questions.

87.3

48.8

42.7

67.9

90.2

84.2

74.0

85.4

62.1

46.0

61.3 development tailored to the needs, experiences and realities of our fellows. It's about fellows taking part in and achieving equitable outcomes, where the product of the engagement is the reduction of gun violence in that community.
Table 2 Advance Peace Sacramento, participants postfellowshipa.

\section{Fellow characteristic}

$\%$ Yes $n$

\section{Alive}

New gun injuries

98.0101

New gun arrest/charge

2.0

86

50

44

70

93

87

76

88

64

47

63

Received assistance for food and/or housing

9.7

Received paid internship or employment

$97.0 \quad 100$

71.8

Reported having a caring adult to talk to, such as an NCA, 84.2

when faced with a difficult situation

Reported peaceful resolution of a conflict that previously $\quad 67.9$

might have resulted in gun use

Rated AP outreach worker one of the most important $\quad 85.4 \quad 88$

adults in life

${ }^{a}$ Not all participants answered all questions.

\section{Reimagining public safety}

While the work of AP is slow-going and seems very individually and locally focused, an underlying thread is to change the public safety systems that for too long have limited opportunities for those growing up in underserved communities. All the NCAs 
recognized that sometimes their work feels like it is just scratching the surface of addressing and eliminating the root causes of trauma in Black communities. One NCA described their work with the fellows as 'keeping fellows clean in a mud puddle,' since they still must go back into neighborhoods, a workforce, a political system, and a culture that does not value Black lives. Yet, just like it took decades of concerted effort on multiple fronts to change the norms of cigarette smoking, AP is building infrastructure and practice for long-term change. They are aware of the structural forces working against them, and part of their institutional and policy change is awareness-raising, writing, and participating in media events, giving public talks, and engaging in policy advocacy.

\section{Conclusions: community-driven healing and justice}

The AP program offers one model for how to acknowledge the traumas of community gun violence and institute practices that help heal and reduce gun crimes. More work is needed to capture the actual impacts of this work on the participants and the practitioners. However, the AP model suggests that communities that have suffered from gun violence are capable of co-creating their own healing strategies with supportive organizations. AP suggests that those who have been traumatized can act as 'wounded healers' and credible messengers to help those most disconnected in communities gain support, love, and hope. Communities hold a wealth of information and valuable assets that can contribute to both locally and culturally relevant healing strategies. AP suggests that community-driven problem-solving can keep locals safe and provide a cascading set of benefits, from direct support to those at the center of violence, to those in their networks to the entire neighborhood and even the city (Norris, 2020). AP offers a model to move beyond just gun violence reduction but to heal, liberate and transform communities.

Received: 12 August 2020; Accepted: 28 April 2021;

Published online: 09 June 2021

\section{References}

Abt T, Winship C (2016) What works in reducing community violence: a metareview and Field Study for The Northern Triangle. USAID. https://www. usaid.gov/sites/default/files/USAID-2016-What-Works-in-ReducingCommunity-Violence-Final-Report.pdf. Accessed 2 May 2020

Akbar M (2017) Urban trauma: a legacy of racism. Purpose Driven Publishing, Hartford

Braga A, Weisburd D, Turchan B (2019) Focused deterrence strategies effects on crime: a systematic review. Campbell Systemat Rev e1051. https://doi.org/ $10.1002 / \mathrm{cl} 2.1051$

Butts J, Roman C, Bostwick L, Porter J (2015) Cure violence: a public health approach. Annu Rev Publ Health 36:39-53. https://doi.org/10.1146/annurevpublhealth-031914-122509

Centers for Disease Control (CDC) (2020). Web-based injury statistics query and reporting system on fatal injury reports, https://www.cdc.gov/injury/wisqars/ fatal.html

Chabria A (2018) The man in charge of Sacramento's new anti-gun violence program was once a shooter himself. Sacramento Bee. https://www.sacbee. com/news/local/article211837849.html

Corburn J, Fukutome A (2019) Advance Peace Stockton: 2018-19 progress report. Institute of Urban \& Regional Development, UC Berkeley

Corburn J, Fututome-Lopez (2020) Outcome evaluation of advance peace Sacramento, 2018-19. https://www.advancepeace.org/wp-content/uploads/2020/ 04/Corburn-and-F-Lopez-Advance-Peace-Sacramento-2-Year-Evaluation03-2020.pdf Accessed 25 Apr 2020

Danese A, McEwen B (2012) Adverse childhood experiences, allostasis, allostatic load, and age-related disease. Physiol Behav 106(1):29-39. https://doi.org/ 10.1016/j.physbeh.2011.08.019

DeCandia CJ, Guarino, K (2015) Trauma-informed care: an ecological response. J Child Youth Care Work 25:7-32. http://acycpjournal.pitt.edu/ojs/jcycw/ article/view/69
Decker MR, Wilcox HC, Holliday CN, Webster DW (2018) An integrated public health approach to interpersonal violence and suicide prevention and response. Public Health Rep. 133(1_suppl):65S-79S. https://doi.org/10.1177/ 0033354918800019

DeCou CR, Lynch SM (2017) Assessing adult exposure to community violence: a review of definitions and measures. Trauma Violence Abuse 18(1):51-61. https://doi.org/10.1177/1524838015590590

Ellis W, Dietz W (2017) A new framework for addressing adverse childhood and community experiences: the Building Community Resilience Model. Acad Pediatr 17(7):S86-S93. https://doi.org/10.1016/j.acap.2016.12.011

Francis MA (2018) Narrative inquiry into the experience of being a victim of gun violence. J Trauma Nurs 25(6):381-8. https://doi.org/10.1097/ JTN.0000000000000406

Friedson M, Sharkey P (2015) Violence and neighborhood disadvantage after the crime decline. Ann Am Acad Political Soc Sci660:341-358. https://doi.org/ $10.1177 / 0002716215579825$

Fullilove M (2004) Root shock: how tearing up urban neighborhoods hurts America, and what we can do about it. Random House, New York

Garvin EC, Cannuscio CC, Branas CC (2013) Greening vacant lots to reduce violent crime: a randomised controlled trial. Injury Prev 19:198-203. https:// doi.org/10.1136/injuryprev-2012-040439

Geller A et al. (2014) Aggressive policing and the mental health of young urban men. Am J Public Health 104(12):2321-27

Gerber MR, Gerber EB (2019) An introduction to trauma and health. In: Gerber M (ed) Trauma-informed healthcare approaches. Springer Nature, Switzerland AG, pp. 3-23

Geronimus AT, Thompson JP (2004) To denigrate, ignore, or disrupt: racial inequality in health and the impact of a policy-induced breakdown of African American communities. Du Bois Rev 1(2):247-279. https://doi.org/10.1017/ S1742058X04042031

Ginwright S (2018) The future of healing: shifting from trauma informed care to healing centered engagement. Medium. https://medium.com/@ginwright/ the-future-of-healing-shifting-from-trauma-informed-care-to-healingcentered-engagement-634f557ce69c Accessed 3 March 2020.

Global Burden of Disease (GBD) (2018) Injury Collaborators, Naghavi M, Marczak LB, et al. 2018. Global mortality from firearms, 1990-2016 JAMA 320 (8):792-814. https://doi.org/10.1001/jama.2018.10060

Hardy KV (2013) Healing the hidden wounds of racial trauma: enhancing professional and research training. Reclaiming Children Youth 22:24-28

Kalesan B, Vyliparambil MA et al. (2019) Cross-sectional study of loss of life expectancy at different ages related to firearm deaths among black and white Americans. BMJ Evid Based Med 24(2):55-8. https://doi.org/10.1136/ bmjebm-2018-111103

Kern L (2016) Rhythms of gentrification: eventfulness and slow violence in a happening neighbourhood. Cult Geogr 23(3):441-457

Lane SD, Rubinstein RA, Bergen-Cico D et al. (2017) Neighborhood trauma due to violence: a multilevel analysis. J Health Care Poor Underserved 28 (1):446-462. https://doi.org/10.1353/hpu.2017.0033

LeBel TP, Richie M, Maruna S (2015) Helping others as a response to reconcile a criminal past: the role of the wounded healer in prisoner reentry programs. Criminal Justice Behav 42(1):108-120

Lowery W, Rich, S (2018) In Sacramento, trying to stop a killing before it happens. Wash Post https://www.washingtonpost.com/investigations/in-sacramentotrying-to-stop-a-killing-before-it-happens/2018/11/08/482be50e-dadd-11e8b732-3c72cbf131f2_story.html

Matthay EC, Farkas K, Rudolph KE et al. (2019) Firearm and nonfirearm violence after operation peacemaker fellowship in Richmond, California, 1996-2016. Am J Public Health 109:1605-1611. https://doi.org/10.2105/AJPH.2019.305288

McEwen C, McEwen BS (2017) Social structure, adversity, toxic stress, and intergenerational poverty: an Early Childhood Model. Annu Rev Sociol 43:445-472. https://doi.org/10.1146/annurev-soc-060116-053252

Motlagh J (2016) A radical approach to gun crime: paying people not to kill each other. UK Guardian https://www.theguardian.com/us-news/2016/jun/09/ richmond-california-ons-gun-crime

Norris T (2020) We keep us safe: building secure, just and inclusive communities. Beacon Press, Boston

Office of Neighborhood Safety (ONS) (2019) Annual Report. Richmond, CA. https://www.ci.richmond.ca.us/271/Office-of-Neighborhood-Safety. Accessed 8 May 2020

Papachristos A, Wildeman C (2014) Network exposure and homicide victimization in an African American community. Am J Public Health 104(1):143-150

Picard-Fritsche S, Cerniglia L (2013) Testing a public health approach to gun violence: an evaluation of Crown Heights Save Our Streets, a replication of the Cure Violence Model. Center for Court Innovation. www.courtinnovation.org/ sites/default/files/documents/SOC_Evaluation.pdf Accessed 29 June 2020

Pressley J, Smith R (2017) No ordinary life: complex narratives of trauma and resilience in under-resourced communities. J Aggress Maltreat Trauma 26(2):137-154 
Rani RS (2017) Building trust cuts violence. Cash also helps. NY Times. https:// www.nytimes.com/2017/02/21/opinion/building-trust-cuts-violence-cashalso-helps.html Accessed 22 Apr 2020

Ranney M, Karb R, Ehrlich P, Bromwich K, Cunningham R, Beidas RS (2019) What are the long-term consequences of youth exposure to firearm injury, and how do we prevent them? A scoping review. J Behav Med. 42 (4):724-40. https://doi.org/10.1007/s10865-019-00035-2

Rivera FP, Studdert DM, Wintemute GJ (2018) Firearm-related mortality: a global public health problem. JAMA. 320(8):764-765. https://doi.org/10.1001/ jama.2018.9942. 2018 Aug 28

Sered D (2019) Until we reckon: violence, mass incarceration and a road to repair. New Press, New York

Skogan WG, Hartnett SM, Bump N, Dubois J (2008) Evaluation of CeaseFire Chicago. http://cureviolence.org/effectiveness/doj-evaluation/. Accessed 12 Apr 2020

Substance Abuse and Mental Health Services Administration (SAMHSA) (2015) SAMHSA's concept of trauma and guidance for a trauma-informed approach. http://store.samhsa.gov/shin/content/SMA14-4884/SMA14-4884.pdf

Wolf AM, Del Prado Lippman A, Glesmann C, Castro E (2015) Process evaluation for the Office of Neighborhood Safety. National Council of Crime and Delinquency, Oakland

Wolfe J, Kimerling R, Brown P, Chrestman K, Levin K (1997) The life stressor checklist-revised (LSC-R) [measurement instrument]. http://www. ptsd.va.gov

\section{Acknowledgements}

This work benefitted from conversations with the Neighborhood Change Agents in all Advance Peace cities, and we appreciate them and their insights.

\section{Competing interests}

The authors declare no competing interests.

\section{Additional information}

Supplementary information The online version contains supplementary material available at https://doi.org/10.1057/s41599-021-00820-y.

Correspondence and requests for materials should be addressed to J.C.

Reprints and permission information is available at http://www.nature.com/reprints

Publisher's note Springer Nature remains neutral with regard to jurisdictional claims in published maps and institutional affiliations.

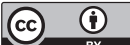

Open Access This article is licensed under a Creative Commons Attribution 4.0 International License, which permits use, sharing, adaptation, distribution and reproduction in any medium or format, as long as you give appropriate credit to the original author(s) and the source, provide a link to the Creative Commons license, and indicate if changes were made. The images or other third party material in this article are included in the article's Creative Commons license, unless indicated otherwise in a credit line to the material. If material is not included in the article's Creative Commons license and your intended use is not permitted by statutory regulation or exceeds the permitted use, you will need to obtain permission directly from the copyright holder. To view a copy of this license, visit http://creativecommons.org/ licenses/by/4.0/.

(C) The Author(s) 2021 